\title{
Adaptive Neuro-Fuzzy Inference System for Prediction of Surgery Time for Ischemic Stroke Patients
}

\author{
Rahma Ali $^{1}$, Uvais Qidwai ${ }^{1 *}$, Saadat K. Ilyas ${ }^{2}$, Naveed Akhtar ${ }^{2}$, \\ Ayman Alboudi ${ }^{3}$, Arsalan Ahmed ${ }^{4}$, Jihad Inshasi ${ }^{3}$
}

\author{
${ }^{1}$ Qatar University, \\ Doha, QATAR \\ ${ }^{2}$ Hamad Medical Corporation, \\ Doha, QATAR \\ ${ }^{3}$ Rashid Hospital, Dubai, UAE \\ ${ }^{4}$ Shifa International Hospital, Islamabad, Pakistan \\ *Corresponding Author
}

DOI: https://doi.org/10.30880/ijie.2019.11.03.007

Received 18 February 2019; Accepted 4 July 2019; Available online 3 September 2019

\begin{abstract}
With the advent of machine learning techniques, creation and utilization of prediction models for different medical procedures including prediction of diagnosis, treatment and recovery of different medical conditions has become the norm. Recent studies focus on the automation of infarction volume growth rate prediction by the utilization of machine learning techniques. These techniques when effectively applied, could significantly help in reducing the time needed to attend to stroke patients. We propose, in this proposal, a Fuzzy Inference System that can determine when a stroke patient should undergo Decompressive Hemic raniectomy. The second infarction volume growth rate and the decision whether a patient needs to undergo this procedure, both predicted outputs of two trained models, act as inputs to this system. While the initial prediction model, that which predicts the second infarction volume growth rate is adopted from an earlier model, we propose the later model in this paper. Three Machine Learn ing techniques - Support Vector Machine, Artificial Neural Network and Adaptive Neuro Fuzzy Inference System with and without the feature reduction technique of Principle Component Analysis were modelled and evaluated, the best of which was selected to model the proposed prediction model. We also defined the structure of Fuzzy Inference System along with its rules and obtained an overall accuracy of $95.7 \%$ with a precision of 1 showing promising results from the use of fuzzy logic.
\end{abstract}

Keywords: Infarction Volume, Neuro-Fuzzy Inference System, Stroke, Infarct Growth Rate, Ischemic Stroke, Support Vector Machine, Artificial Neural Network

\section{Introduction}

There is a worldwide trend in the increase of the number of stroke cases reported every year. According to the World Health Organization, stroke is one of the leading causes of death in 2016 with approximately 5.8 million deaths reported in 2016 alone. While a stroke could happen to anyone, multiple risk factors such as age, hypertension, obesity and diabetes contribute significantly to its occurrence. 
Stroke, also known as brain-attack (similar to heart attack), is of two main types - Ischemic and Haemorrhagic. Ischemic strokes occur when blood clots block brain arteries. This leads to infarction, a condition in which the brain cells end up dying due to the blockage since they are deprived of blood that is necessary for oxygen and nutrients transportation. Consequently, brain edema or swelling of the brain starts to occur due to the chemical imbalance caused in that area. If no immed iate action is taken to address this and given that the skull cannot expand, the built-up pressure will then be directed inwards towards the brain stem. This may result in the development of physical disabilities and in worst case scenarios may even cause death. Thus, early diagnosis and therefore treatment of stroke is of paramount importance.

Multiple works in the literature address prediction of stroke and its corresponding diagnosis using Machine Learn ing (ML) Techniques [1], [2], [3]. The proposed solution proposed in [4] is one such example. The ir proposed solution predicts the second infarction volume growth rate using several clinical features such as age, HbA1c reading among others. In addition to that, it also considers first infarction volume growth rate as input. While it is possible to effectively predict the infarction volume of a stroke patient, it can nevertheless be an immensely dispersed scenario. This is due to the fact that it not only depends upon the patients' current conditions but also on their conditions at the time at which the CT scans were performed. This in its self is another variable with a big variance connected to it. Interestingly, it has been found in [5] that the rate of volumetric growth of an infarction growth remains fairly consistent for a sizeable time duration, and any volume can be predicted with reasonable accuracy within a predetermined set time span.

In our proposed solution, we wish to extend the work done in [4]. In order to achieve this, we will first model a mach ine learning model that predicts to determine whether a patient needs to be operated upon. The predicted output of this model and that of baseline prediction model will then be fed into a fuzzy Inference System that will determine when the patient needs to be operated upon. We hope this will offer a more helpful and complete solution to the medical professionals and aid them in their decision-making process, in particular their ability to determine when a patient needs to undergo surgery.

\section{Related Work}

Utilization of machine learning techniques in the prediction of medical conditions is not new. A lot of work has already been done in the literature in this regard. Accordingly, the use of ML techniques in the prediction of various aspects of stroke patients is also not new. Of these, Support Vector Machine (SVM) and neural networks are the most popular techniques used in medical applications [6], [7]. Several works such as those discussed in [8], [9], [10] only use medical images such as CT scans in order to train their models. Yet others, such as those discussed in [11], [12], use a combination of med ical images along with other clinical data in order to train the model. However, works that use only clinical data such as proposed in our work are very limited in nu mber. This limitation is further confirmed in the survey paper of Jiang et. al [6] in which they discuss how most of the papers in the literature use ML and deep learning based techniques for medical image analysis more than they do for medical data analysis. For in stance, one such work that uses ML on only medical data is found in the work proposed by Asadi et. al [13]. In this they modelled and compared two supervised machine learning techniques - SVM and Artificial Neural Network (ANN) for stroke analysis. Their models considered multiple inputs, including risk factors of the patients to predict the expected outcome of endovascular intervention performed on patients with acute anterior circulation ischaemic stroke in terms of mRS score. They trained, validated and tested their models on randomly divided data from a dataset of 107 records. They concluded that SVM had a better performance, when compared with the performance of ANN. The overall precision of their proposed system was $87 \%$ while their model accuracy was approximately $70 \%$. On the other hand, an example of prediction model that combines medical numerical data along with medical image data is that proposed by Bentley et. al [11]. In this, they trained an SVM model that would take in, as input, raw CT scan images along with other clinical information and as output predict if the stroke patient is at risk of Symptomatic Intracerebral Haemorrhage, a complication that affects patients who are treated by intravenous thrombolysis. To validate their work, they used k-fold cross-validation by splitting their dataset into 106 and 10 training and testing sets respectively for over 1760 repetit ions. They compared their work to the outcome of another work that used radiologists' interpretations of the CT scan images (along with clinical variables) in their model, rather than using raw images and found theirs to outperform the latter's in terms of predictive performance. Another version of inputs is that in which they consider symptoms along with numerical inputs as proposed by Mirtskhulava et. al [14]. They modelled a neural network model having two hidden layers which accepted 16 inputs to predict the risk of stroke in the examined patient. These inputs included a combination of patient symptoms and stroke related risk factors. Their proposed system had a binary output which predicted whether or not the patient would suffer a stroke. Therefore, an output of one meant the patient is at a risk of stroke, while zero meant otherwise.

There are also other proposed systems that utilize Adaptive Neuro- Fu zzy Inference System as their underlying system. One such work is that proposed in [5]. In this work, they experimented with using Adaptive Neuro- Fuzzy Inference System (ANFIS), an algorithm that combines Artificial Neural Network (ANN) and Fu zzy Inference System (FIS) to investigate infarction growth pattern and use that to predict infarction growth rate and infarction volume at a given instance for stroke patients. They focused on patients that had large vessel occlusion in their anterior circulation. 
Prediction using this method has not been reported previously and appeared to offer promising results when compared with other models used in the literature. Their proposed solution was able to predict the infarction growth rate and infarction volume of the third CT scan without any statistical difference when compared to the ground truth $(\mathrm{P}=$ 0.489).

Others use a variation to using straightforward ML techniques by applying feature reduction techniques such as Principal Component Analysis (PCA) to the dataset to enhance the resulting prediction models. Proposed solutions such as those proposed in [15], [16], [17] perform feature reduction using the technique of PCA then predict the output from the resulting reduced dataset by utilizing ANFIS. By doing so, they state that their prediction models are able to perform better prediction than most contemporary machine learning prediction models for the same problem. The baseline of our proposed solution is one such work that does it for stroke-based predictions. The proposed solution is based on an improved version of [5] where in feature reduction is initially performed on the dataset and then the second infarction growth rate is predicted from the reduced dataset using ANFIS. The ir proposed solution obtained a RMSE of 0.196 which is $55.35 \%$ improvement to the original obtained RMSE without PCA.

For our proposed solution, we intend to model a Fuzzy Inference System that predicts when a patient needs to undergo Decompressive Hemic raniecto my. The inputs to this system are the pred icted output from the baseline solution and the predicted output of a model trained to predict whether a patient needs to undergo the surgery. Similar to [4], which our work is an extension of, we want to prove that we can use FIS to accurately predict for an argumentatively unique and significantly complicated problem than those already addressed in the literature by others. Complication occurs due to the fact that unlike in most other medical conditions already addressed in the literature that usually have a larger sized offline dataset available to model their proposed solution on, the dataset available for stroke is on the other hand very limited. Also unlike in other medical problems as those discussed above that deal with diagnosis or detection of a medical condition that can be done over a period of time, prediction of infarction volume and thus the decision to operate on a patient, on the other hand, is very time critical. These decisions need to be taken as accurately and as fast as possible to avoid further health complications such as disabilities or death. By building an effective prediction model, we offer to not only significantly reduce the decision time required by doctors but also help reduce the number of CT scans required by patients to only one.

The rest of the paper is structured as follows - A brief explanation of the underlying theory of the techniques utilized in the proposed solution are briefly explained in Section 3 with Subsection 3.1 focusing on Principal Component Analysis, and Subsection 3.2 summarizing Support Vector Machine, one of the Machine Learning techniques modelled and evaluated to create the prediction model. Similarly, Section 3.3 offers a brief conceptual description of Artificial Neural Network while Section 3.4 describes Adaptive Neuro Fuzzy Inference System. Section 4 reviews the dataset used while Section 5 explains the methodology adopted for implementing our proposed system. The evaluation metrics used to evaluate our proposed system and the results obtained are then discussed in Section 6 before concluding in Section 7.

\section{Theory}

\subsection{Principal Component Analysis}

One of the major issues with medical datasets is that they predominately consist of a set of imbalanced output classes [18], [19], [20]. Other issues pertaining to them is the presence of unfavorable feature to observation ratio, which usually occur when a small dataset has many features. This results in a training model that is not able to effectively learn resulting in a classifier that performs poorly [21]. Due to this, techniques, called feature reduction techniques, that reduce the number of features without significantly minimizing the information contained within them are used to overcome is sues pertaining to unbalanced datasets. They do this by finding patterns in high dimensional datasets. An example of one such technique is the statistical technique of Principal Component Analysis (PCA). PCA is based on the assumption that most information of the dataset is covered in the directions where the variations of the dataset are largest. Therefore to perform feature reduction, it creates standardized linear projections, called principal components, that are linear combinations of the orig inal features and are directed at the directions where most variation of the data exists, thereby reducing number of variables [22]. Depending on how much variation needs to be preserved, the appropriate subspace covered by these principal components is selected [16]. When applied to medical datasets, PCA helps improve the generated training model. Another strength of PCA is in its ability to define the space of spread of the overall data. This helps in detecting and eventually eliminating outliers [23], a necessity in medical settings.

\subsection{Support Vector Machine}

There are two main types of Support vector machines (SVM): support vector classification (SVC) and support vector regression (SVR). SVM, being a discriminative classifier, is formally defined by one or more separating hyperplanes. Given a labelled training data, the SVM algorithm outputs the most optimal set of hyperplanes that best categorizes the new samples. For a two-dimensional space, considered a linear based separation, the hyperplane is defined as the line dividing a plane into two parts where each class lie on the either side of the line. SVM is popularly used in multiple domains from image recognition to text classification to bioinformatics, and so on. Its strength lies in 
its ability to offer robust performance in less adequate scenarios such as those involving sparse or noisy data. This makes SVM the ML technique of choice in many applications [23]. Initially developed to classify training data without errors, SVM was extended to support classifying training data with errors making it as powerful and applicable as neural networks [24]. Add to this, SVM can also handle nonlinear classification effectively. It does this by using a nonlinear kernel function to map samples from low dimensional input space to high dimensional feature space [25]. Another version of SVM, meant for regression also exists. The performance of this version of SVM crucially depends on the shape of the kernel function and other hyper-parameters that epitomize the noise distribution characteristics in the training data. In the case of any SVM classifier, the model generated by a classifier only depends on a subset of training data due to the fact the cost function of the model does not consider training points beyond the marg in. However, the reason for a SVM regression does so because the cost function ignores any training data close within a certain threshold to the model prediction [26]. In this proposed solution, we utilized SVM in both classification and regression prediction as explained in further sections.

\subsection{Artificial Neural Network}

Artificial Neural Network (ANN) is inspired by the functioning of the human brain and replicates the way humans learn. It is a ML technique that comprises of a set of networks called neural networks which consist of input and output layers as well as a hidden layer that transform input to output. While the concept of neural networks or perceptrons have been around for a very long time now, only recently have their use become a major part of artificial intelligence. This is mainly due to the recent existence of the concept of backpropagation. Backpropagation allows the networks to readjust the hidden layers of neurons in situations where the output is not as expected thereby strengthening the learning and therefore prediction ability of the training model.

The architecture of a typical ANN can be visualized as weighted directed graphs with artificial neurons as nodes and directed and weighted edges as connections between the output and input neurons. When considering the connections, ANN can be characterized into two categories - feedforward networks and feedback (recurrent) networks. In feedforward based ANNs, the graphs represented by the connections have no loops. While those in feedback networks have loops due to occurrence of feedback connections. The output from a feedforward network is static since they produce only one set of output values rather than a sequence of output values from a given input with no feedback correction option. This makes feedback network memoryless given their response to input does not depend on the previously modified network state. Feedback networks on the other hand are dynamic systems since new neuron outputs are generated when a new input pattern is presented and given, they possess feedback paths, the input to each neuron can then be modified resulting a new network state [27]. In this paper, we only considered feedforward ANNs.

\subsection{Adaptive Neuro Fuzzy Inference System}

Fuzzy logic is a computing approach that is based on degree of truth fulness rather than crisp true or false values, commonly known as Boolean logic, which modern computers are based on. The use of Fuzzy logic is highly recommended in modelling scenarios that are characterized as inherently imprecise or vague. This is due to their ability to consider this vagueness in their modelling. However, the main issue with Fuzzy logic is that there exists no systematic procedure for the design of a fuzzy logic controller, making it hard to implement an accurate and representative model. Due to this and given that neural networks on the other hand have the ability to learn from the environment, self-organize their structure and adapt to it in an interactive manner as a result, incorporating them with a fuzzy logic controller with neural network makes more sense [28]. ANFIS is one such methodology that combines these two. By having an adaptive neuro fuzzy system that can self-organize its structure according to the environment, we are able to combine the advantages of both systems.

As mentioned earlier, ANFIS is a machine learning technique, the core of which is an ANN based on TakagiSugeno Fuzzy Inference System. It is implemented by an underlying framework of adaptive networks. One of the advantage of using ANFIS is its ability to allow fuzzy rules to be extract from numerical data or expert knowledge and adaptively construct a rule base on that [28]. Because it uses a hybrid learning procedure, it is able to construct an input-output mapping based on human knowledge, done using fuzzy if-then rules and input-output data pairs [28], [29]. It is in this if-then rules where the medical knowledge of the medical practitioners can be included. Thus, ANFIS combines neural networks along with injected expert heuristics to build a model.

The architecture and the learning procedure of ANFIS is grounded on adaptive network which is a feedforward based neural network with supervised learning capability. It consists of nodes and directional links that connect these nodes. Some or all of these nodes could be made adaptive thus making the outputs depend on the parameters belonging to these nodes. The learning rules define how these parameters are changed to reach a predefined minimum error. It is therefore due to this customization, learning and relearning by going through multiple passes and its consideration of expert knowledge that makes ANFIS an applicable machine learning option in the medical domain. It is also due to these reasons one would expect it would produce a more realistic functioning model. 


\section{Dataset}

As previously mentioned, this work is an extension to an earlier work [4]. As a result, we utilized the same dataset as used in the prior work albeit only considering 117 records of the already utilized 122 records. The reduction in the number of records was to overco me the null values that were introduced by the new included outputs. In addition to that, since treatment time, considered an input in [4], is considered as an output to our proposed solution, we have removed it as possible input and this resulted in only considering 14 features. As mentioned in [4], this dataset consists of patient data of patients that had three computed tomography (CT) scans of their brains performed and found to have evidence of acute ischemia. They belong to a pooled Decompressive Hemicraniectomy database, the constituents of which were obtained from three referral centers in three different countries namely Qatar, UAE and Pakistan. These selected 14 features provide relevant medical details of the selected 117 stroke patients. These medical details include patient age, their diastolic and systolic blood pressure readings, among others. In addition to these, the initial In farction volume rate was also considered as an input to both the models that are generated. A detailed description of the features present in the dataset and their respective median and standard deviation values are presented in Table 1.

Table 1 - Features included in the dataset

\begin{tabular}{|c|c|c|c|}
\hline Feature & Description & Value & Median \pm Standard Deviation \\
\hline Age & - & Presented In years & $50 \pm 12.641$ \\
\hline HTN & $\begin{array}{l}\text { Hypertension } \\
\text { diagnosis }\end{array}$ & $\begin{array}{l}0 \text { - Absent } \\
1 \text { - Present }\end{array}$ & $1 \pm 0.500$ \\
\hline DBP & Diastolic blood pressure reading & - & $76.5 \pm 8.798$ \\
\hline SBP & $\begin{array}{l}\text { Systolic blood } \\
\text { pressure reading }\end{array}$ & - & $138 \pm 13.441$ \\
\hline DM & Diabetes diagnosis & $\begin{array}{l}0 \text { - Absent } \\
1 \text { - Present }\end{array}$ & $0 \pm 0.495$ \\
\hline HBA1C & HBA1C reading & 5.4 to 16.5 & $2 \pm 0.870$ \\
\hline $\begin{array}{l}\text { Ejection } \\
\text { Fraction }\end{array}$ & $\begin{array}{l}\text { Time taken to pull out the blood } \\
\text { clot. }\end{array}$ & In minutes & $50 \pm 9.929$ \\
\hline DYS & Dysplasia diagnosis & $\begin{array}{l}0 \text { - Absent } \\
1 \text { - Present }\end{array}$ & $0 \pm 0.500$ \\
\hline $\begin{array}{l}\text { Clot } \\
\text { Burden } \\
\text { Score }\end{array}$ & $\begin{array}{l}\text { This score represents how much } \\
\text { pressure the clot will be causing in } \\
\text { the brain area where it is blocking } \\
\text { the flow. The score depends upon } \\
\text { length, density, volume, location, } \\
\text { twisted or straight and chemical } \\
\text { level. }\end{array}$ & $0-10$ & $8 \pm 1.494$ \\
\hline METS & $\begin{array}{l}\text { Metabolic Syndrome }- \text { a } \\
\text { combination of multiple } \\
\text { conditions that increase the risk of } \\
\text { stroke and that are combined into } \\
\text { one score. }\end{array}$ & $\begin{array}{l}0-\text { All absent } \\
1 \text { - At least one } \\
\text { present }\end{array}$ & $1 \pm 0.500$ \\
\hline $\begin{array}{l}\text { Collateral } \\
\text { Score }\end{array}$ & $\begin{array}{l}\text { Temporary blood vessels that } \\
\text { replace the blocked blood vessel. }\end{array}$ & $0-3$ & $2 \pm 0.825$ \\
\hline
\end{tabular}


$\begin{array}{lll}\text { Smoking States if the patient is a smoker or } & \begin{array}{l}0 \text { - Non-smoker } \\ \text { not. }\end{array} & 0 \pm 0.405\end{array}$

\begin{tabular}{|c|c|c|}
\hline $\begin{array}{l}\text { Modified } \\
\text { TICI }\end{array}$ & $\begin{array}{l}\text { A score that determines the } \\
\text { response of thrombolytic therapy } \\
\text { for ischaemic stroke. }\end{array}$ & $0-3$ \\
\hline
\end{tabular}
for ischaemic stroke.

These features were used to create 2 ML models - infarction volume prediction model and decision for surgery prediction model to output the predicted second infarction growth rate and whether the patient needs to undergo a surgery or not.

\section{Methodology}

The entire proposed system was developed in MATLAB. The methodology adopted will be discussed in this section which includes data preparation by pre-processing the dataset, performing additional feature reduction on the dataset and then finally the steps taken to model both the proposed prediction model and the FIS.

\subsection{Pre-processing}

\subsubsection{Null Removal and Normalization}

The first step in the adopted methodology of our proposed solution was to remove any records that contained any null values. The dataset was then normalized by normalizing each column individually.

\subsubsection{Training and Testing Set Generation}

Once the complete dataset was normalized, it was then split into training and testing set each of which consisted of indices picked at random. This random selection of training and testing set of indices was performed 100 times. The most statistically similar training and testing sets were selected from these 100 sets. Our definition of statistically similar was that the corresponding columns in the training and testing sets had the least difference in terms of standard deviation and median. By opting for statistically similar testing and training sets, we attempted to create a more representative training and testing sets thereby setting up a more accurate evaluation of the training models generated. This thus resulted in a randomly split yet statistically similar training and testing sets. $70 \%$ instances of the original dataset were used for training, while $30 \%$ of it was used for testing.

\subsection{Model Creation}

Once we preprocessed the data and prepared the training and testing set, the next step was to prepare the two ML prediction models - IVGR2 prediction modeled as presented in [4] and our proposed prediction model that would predict whether a patient would need to undergo surgery. In addition to that we also formulated the rules of the FIS. The FIS would determine the time of surgery based on the predicted outputs of the before mentioned models and the defined rule set.

\subsubsection{Proposed Prediction Model}

We primarily implemented our proposed prediction model in 3 machine learning models - SVM, ANFIS and ANN. We then applied feature reduction technique of PCA to these models which resulted in 6 prediction models. The performance of each of these models is discussed in elaboration in Section 6. The best performing model - SVM - was selected and its prediction output along with the output of the baseline prediction model were fed into the FIS. 


\subsubsection{Fuzzy Inference System}

We then implemented the FIS by specifying the rule set for the inference system. Before defining the rules, we first defined the membership functions for the inputs. For IVGR2, we selected Gaussian membership functions to represent the predicted infarction volumes and labelled small, medium and large. Similarly, for the predicted output values of the second model that which represented the decision for surgery (DHCN), we used trapezoidal shaped membership functions. The inputs were classified into Surgery or No Surgery (NoSurgery). The output, time of surgery (Time OfSurgery), was split into three categories - No Surgery Required (NoSurgery), Surgery Required in 48 hours (Surgery $48 \mathrm{Hr}$ ) and Surgery Required in 24 hours (Surgery24Hr). We utilized triangular-shaped membership function for the output. Once we defined these, we outlined the rules as follows -

1. If (IVGR2 is Small) and (DHCN is NoSurgery) then (TimeOfSurgery is NoSurgery)

2. If (IVGR2 is Medium) and (DHCN is Surgery) then (TimeOfSurgery is Surgery48Hr)

3. If (IVGR2 is Large) and (DHCN is NoSurgery) then (TimeOfSurgery is Surgery48Hr)

4. If (IVGR2 is Large) and (DHCN is Surgery) then (TimeOfSurgery is Surgery24Hr)

In the next section we evaluate the generated models and system.

\section{Experimental Results and Discussion}

In order to evaluate our work, we selected multiple evaluation metrics for both regression-based prediction model and binary based classification model. Given infraction volume prediction is a regression problem, we evaluated our proposed solution using regression appropriate evaluation techniques such as Root Mean Square error (RMSE), Mean Absolute Error (MAE) and R-Squared $\left(\mathrm{R}^{2}\right)$. Like wise, since determining whether a stroke patient requires a surgery or not is a binary classification problem, we utilized evaluation techniques such as Accuracy, Precision and Recall. In this section, we will describe and discuss the different evaluation metrics and the results obtained for each of them.

RMSE, which is the square root of the Mean Square Error (MSE), is a popular technique used as an evaluation metric in the literature. The reason we selected this measure was because it empowers large number deviations and when compared to MSE gives higher weightage and thereby punishes large errors more. This is critical in our solution since we are modelling a prediction model for a medical setting and this involves predicting health related outcomes. Therefore, utmost accuracy must be obtained while avoiding any minute errors as much as possible. The formu la RMSE is as shown below.

$$
R M S E=\sqrt{\frac{1}{N} \sum_{i=1}^{N}\left(\text { actual }_{i}-\text { predicted }_{i}\right)^{2}}
$$

Another measure we used to evaluate the regression prediction model was the MAE. It measures the mean value of error in a set of observations without considering their direction. The score averages over the absolute differences of a set of target and predicted observation, while giving equal weights to all individual differences. The equation of MAE is as shown below.

$$
M A E=\frac{1}{N} \sum_{i=1}^{N} \mid \text { actual }_{i}-\text { predicted }_{i} \mid
$$

Since we extended an earlier work [4] in which a combination of ANFIS and PCA is used to model the IVGR2 prediction model, we modelled the same model and evaluated it on the before mentioned metrics. The results obtained can be seen in Table 2. From this we can deduce that the predicted outputs for the better performing model - ANFIS with PCA - has a 15\% RMSE and a MAE of $12 \%$ when compared to the target output.

Table 2 - Evaluation results of IVGR2 prediction model

\begin{tabular}{lll}
\hline ML Model & RMSE & MAE \\
\hline ANFIS & 0.250 & 0.162 \\
ANFIS with PCA & $\mathbf{0 . 1 5 0}$ & $\mathbf{0 . 1 1 8}$ \\
\hline
\end{tabular}

On the other hand, accuracy, precision and recall were used to evaluate both the decision for su rgery prediction model and the final proposed FIS. Accuracy is defined as the fraction of predictions the model being evaluated got 
correct. Precision is the fraction of observations that are predicted positive and are actually positive. Lastly, recall calculates how many actual positives the model labels positive. We evaluated all the 6 models and the results are as show in Table 3. We obtained the best results with ANFIS when feature reduction of PCA is applied.

Table 3 - Evaluation results of decision for surgery prediction model

\begin{tabular}{llll}
\hline ML Model & Accuracy & Precision & Recall \\
\hline SVM & 0.915 & 1 & 0.915 \\
SVM with PCA & 0.915 & 1 & 0.915 \\
& & & \\
ANFIS & 0.872 & 0.884 & 0.974 \\
ANFIS with PCA & $\mathbf{0 . 9 7 8}$ & $\mathbf{1}$ & $\mathbf{0 . 9 1 4}$ \\
ANN & 0.829 & 0.860 & 0.948 \\
& & & 0.946 \\
ANN with PCA & 0.787 & 0.814 & \\
\hline
\end{tabular}

Table 4 below shows the confusion matrix obtained by the prediction results of FIS.

Table 4 - Confusion matrix of FIS

\begin{tabular}{cccc}
\hline & \multicolumn{3}{c}{ Predicted Output } \\
\hline & & 0 & 1 \\
Actual Outputs & 0 & 45 & 0 \\
& 1 & 2 & 0 \\
\hline
\end{tabular}

The number of true positive is at 45 , resulting at an accuracy of $95.7 \%$. However as much as it is important for a system to have high accuracy; for our problem, it is more important that our system is always able to make the right decision, which is the essence of precision. As shown in Table 5, the precision of the FIS is at 1 which is $100 \%$. Thus, our proposed solution performs well for the situation at hand.

Table 5 - Obtained evaluation results of overall system

\begin{tabular}{ccc}
\hline Accuracy & Precision & Recall \\
\hline & & \\
0.957 & 1 & 0.957
\end{tabular}

The generated 3D surface of the FIS is as shown in Fig. 1.

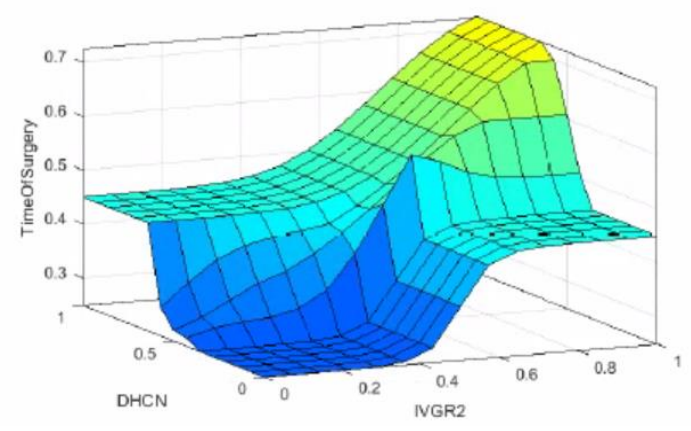

Fig. 1 - 3D Decision Surface of Generated FIS Inputs vs Output 


\section{Conclusion}

In this work we proposed a system that implements a FIS to determine when an ischemic stroke patient needs to undergo Decompressive Hemicran iectomy. This decision is based on the output of two prediction models that predict the IVGR2 of the patient and the decision if the patient needs to undergo a surgery or not. Our evaluation results show that the prediction outcome of our proposed solution performs significantly well obtaining an accuracy of approximately $96 \%$.

\section{References}

[1] B. Letham, C. Rudin, T. H. McCormick, and D. Madigan, "Interpretable classifiers using rules and Bayesian analysis: Building a better stroke prediction model,” Ann. Appl. Stat., vol. 9, no. 3, pp. 1350-1371, Sep. 2015.

[2] T. Lumley, R. A. Kronmal, M. Cushman, T. A. Manolio, and S. Goldstein, "A stroke prediction score in the elderly: validation and Web-based application," 2002.

[3] A. Khosla, Y. Cao, C. C.-Y. Lin, H.-K. Chiu, J. Hu, and H. Lee, "An integrated machine learning approach to stroke prediction," in Proceedings of the 16th ACM SIGKDD international conference on Knowledge discovery and data mining - KDD '10, 2010, p. 183.

[4] R. Ali, U. Qidwai, and S. K. Ilyas, "Use of Combination of PCA and ANFIS in Infarction Volume Growth Rate Prediction in Ischemic Stroke," in 2018 IEEE-EMBS Conference on Biomedical Engineering and Sciences (IECBES), 2018, pp. 324-329.

[5] S. Kamran et al., "Prediction of infarction volume and infarction growth rate in acute ischemic stroke," Sci. Rep., vol. 7, no. 1, p. 7565, Dec. 2017.

[6] F. Jiang et al., "Artificial intelligence in healthcare: Past, present and future," Stroke Vasc. Neurol., vol. 2, no. 4, pp. 230-243, 2017.

[7] J. T. Senders et al., "Machine Learning and Neurosurgical Outcome Prediction: A Systematic Review," World Neurosurg., vol. 109, p. 476-486.e1, 2018.

[8] A. Lisowska et al., "Context-Aware Convolutional Neural Networks for Stroke Sign Detection in Non-contrast CT Scans," Springer, Cham, 2017, pp. 494-505.

[9] Y. Wang, A. K. Katsaggelos, X. Wang, and T. B. Parrish, "A deep symmetry convnet for stroke lesion segmentation," in 2016 IEEE International Conference on Image Processing (ICIP), 2016, pp. 111-115.

[10] Y. Choi, Y. Kwon, H. Lee, B. J. Kim, M. C. Paik, and J.-H. Won, "Ensemble of Deep Convolutional Neural Networks for Prognosis of Ischemic Stroke," Springer, Cham, 2016, pp. 231-243.

[11] P. Bentley et al., "Prediction of stroke thrombolysis outcome using CT brain machine learning," NeuroImage Clin., vol. 4, pp. 635-640, 2014.

[12] J. Hampton-Till et al., "AUTOMATED QUANTIFICATION OF STROKE DAMAGE ON BRAIN COMPUTED TOMOGRAPHY SCANS : e-ASPECTS,” Eur. Med.J., vol. 3, no. 1, pp. 69-74, 2015.

[13] H. Asadi, R. Dowling, B. Yan, and P. Mitchell, "Machine learning for outcome prediction of acute ischemic stroke post intra-arterial therapy," PLoS One, vol. 9, no. 2, pp. 14-19, 2014.

[14] L. Mirtskhulava, J. Wong, S. Al-Majeed, and G. Pearce, "Artificial Neural Network Model in Stroke Diagnosis," Proc. - UKSim-AMSS 17th Int. Conf. Comput. Model. Simulation, UKSim 2015, pp. 50-53, 2016.

[15] E. Avci and I. Turkoglu, "An intelligent diagnosis systembased on principle component analysis and ANFIS for the heart valve diseases," Expert Syst. Appl., vol. 36, no. 2, pp. 2873-2878, Mar. 2009.

[16] K. Polat and S. Güneş, “An expert systemapproach based on principal component analys is and adaptive neurofuzzy inference systemto diagnos is of diabetes disease," Digit. Signal Process., vol. 17, no. 4, pp. 702-710, Jul. 2007.

[17] K. Polat and S. Güneş, "Automatic determination of diseases related to lymph systemfrom lymphography data using principles component analys is (PCA), fuzzy weighting pre-processing and ANFIS," Expert Syst. Appl., vol. 33, no. 3, pp. 636-641, Oct. 2007.

[18] M. M. Rahman and D. N. Davis, "Addressing the Class Imbalance Problem in Medical Datasets," Int. J. Mach. Learn. Comput., vol. 3, no. 2, pp. 224-228, 2013.

[19] L. Mena and J. A. Gonzalez, "Machine Learning for Imbalanced Datasets: Application in Medical Diagnostic."

[20] P. Yang, L. Xu, B. B. Zhou, Z. Zhang, and A. Y. Zomaya, "A particle swarm based hybrid systemfor imbalanced medical data sampling," BMC Genomics, vol. 10, no. Suppl 3, p. S34, Dec. 2009.

[21] M. A. Mazurowski, P. A. Habas, J. M. Zurada, J. Y. Lo, J. A. Baker, and G. D. Tourassi, "Training neural network classifiers for medical decision making: The effects of imbalanced datasets on classification performance," Neural Networks, vol. 21, no. 2-3, pp. 427-436, Mar. 2008.

[22] I. Babaoğlu, O. Fındık, and M. Bayrak, "Effects of principle component analysis on as ses sment of coronary artery diseases using support vector machine," Expert Syst. Appl., vol. 37, no. 3, pp. 2182-2185, Mar. 2010.

[23] T. S. Furey, N. Cristianini, N. Duffy, D. W. Bednarski, M. Schummer, and D. Haussler, "Support vector 
machine classification and validation of cancer tissue samples using microarray expression data," Bioinformatics, vol. 16, no. 10, pp. 906-914, 2000.

[24] C. Corinna and V. Vladimir, "Support-Vector Networks," Mach. Learn., vol. 20, no. 3, pp. 273-297, 1995.

[25] J. Xie and C. Wang, "Using support vector machines with a novel hybrid feature selection method for diagnosis of erythemato-squamous diseases," Expert Syst. Appl., vol. 38, no. 5, pp. 5809-5815, 2011.

[26] B. Debasish, S. Pal, and D. Chandra Patranabis, "Support Vector Regression," Neural Inf. Process. - Lett. Rev., vol. 11, no. 10, pp. 203-224, 2007.

[27] A. K. Jain, J. Mao, and K. M. Mohiuddin, "Artificial neural networks: A tutorial," Computer (Long. Beach. Calif)., vol. 29, no. 3, pp.31-44, 1996.

[28] F. Chang and Y. Chang, "Adaptive neuro-fuzzy inference systemfor prediction of water level in reservoir," vol. 29, pp. 1-10, 2006.

[29] J.-S. R. Jang, “ANFIS: adaptive-network-based fuzzy inference system,” IEEE Trans. Syst. Man. Cybern., vol. 23, no. 3, pp. 665-685, 1993. 